Communications in Physics, Vol. 26, No. 4 (2016), pp. 369-379

DOI:10.15625/0868-3166/26/4/9117

\title{
HIGGS MASS CONSTRAINT ON A LOW SCALE SUSY MODEL
}

\author{
TRAN MINH HIEU ${ }^{1, \dagger}$, NGUYEN THU HUONG ${ }^{2}$ AND TRIEU QUYNH TRANG ${ }^{3}$ \\ ${ }^{1}$ Hanoi University of Science and Technology \\ ${ }^{2}$ VNU University of Science, Vietnam National University, Hanoi \\ ${ }^{3}$ Nam Dinh Teacher's Training College \\ ${ }^{\dagger}$ E-mail: hieu.tranminh@hust.edu.vn
}

Received 01 January 2017

Accepted for publication 18 February 2017

\begin{abstract}
Randal-Sundrum models have an interesting feature that an effective physical scale can be generated from a much larger one of the underlying theory. In this paper, we investigate a model of supersymmetry in the Randall-Sundrum spacetime with a low cutoff scale. Due to the warp factor, the Kaluza-Klein scale is warped down to $\mathscr{O}(100) \mathrm{TeV}$. With the MSSM superfields living in the bulk, the soft SUSY breaking terms of the $4 D$ effective theory are derived from the original 5D Lagrangian by integrating out the extra-dimension. We examine the constraint of the Higgs boson mass measurement on the model. As a result, the viable parameter space with the ability to reproduce a $125 \mathrm{GeV}$ Higgs mass is identified. The constraint rules out parameter regions with the stop masses larger than $20 \mathrm{TeV}$. Therefore, the parameter space of the model can be explored in a future $100 \mathrm{TeV}$ collider.
\end{abstract}

Keywords: Higgs boson, supersymmetry, Randall-Sundrum, extra-dimension.

Classification numbers: 11.30.Pb, 12.60.-i..

\section{INTRODUCTION}

The discovery of a Higgs boson [1] by the ATLAS and CMS Colaborations at the Large Hadron Collider (LHC) has fulfilled the prediction of the standard model (SM). However, it also raises many questions that have challenged the SM for a long time. To address these questions, physics beyond the SM is necessary. One of the long-standing problem is the so-called gauge hierarchy problem. In a theory with a scalar field like the Higgs boson, the electroweak scale becomes unstable due to quantum corrections which are determined by the mass scale of an underlying theory (such as the grand unification scale, or the Planck scale).

(C)2016 Vietnam Academy of Science and Technology 
A famous solution to the hierarchy problem comes from the supersymmetry (SUSY) which is a special symmetry between two kinds of particle with different spins, fermion and boson. The contributions of these superpartners exactly cancel all the quadratic divergences in loop corrections, making the theory ultra-violet (UV) insensitive. Because superpartners cannot have the same masses as the corresponding SM particles, the SUSY must be broken spontaneously. Therefore, a SUSY breaking mechanism is required. Among several possibilities, we are interested in the gravity mediation mechanism since gravitation is a universal interaction that acts on any kind of matter. In SUSY breaking models, the gravity mediation appears effectively as Planck-suppressed high dimensional operators. Beside traditional 4D models, 5D SUSY have been also studied in the context of spacetime with an extra dimension [2]. Such models provide rich phenomenology and interesting predictions [3].

In this paper, we focus on a setup of the minimal SUSY extension of the SM (MSSM) in the 5D spacetime where the extra dimension is warped and compactified on an $S_{1} / Z_{2}$ orbifold [4]. This orbifold possesses two fixed points where two 3-branes (ultra-violet (UV) and infra-red (IR) ones) locate. This background geometry was originally proposed by Randall and Sundrum (RS) in an attempt to solve the hierarchy problem [5], however it cannot explain the existence of dark matter. In our study, such problems are addressed by the SUSY, while the RS background plays an essential role to lower the cutoff scale and provides a landscape for explaining the diversity of masses and couplings [4]. Our model has a hidden sector confined on the UV brane, and all the MSSM superfields live in the entire 5D spacetime. The 4D effective soft SUSY breaking terms are calculated from the underlying 5D Lagrangian. Many phenomenological models have been found to be strongly restricted [6] after taking into account the measurement of the Higgs boson mass at the LHC. Here we investigate this constraint on the considered model, and identify the viable portion of the parameter space that can reproduce the LHC result on the SM-like Higgs boson mass.

The structure of the paper is as follows. In Section 2, we present the model setup with a 5D Lagrangian, and the result of effective soft SUSY breaking terms in 4D effective theory. In Section 3, the implication of the Higgs mass measurement on the model is studied, and the viable parameter space is identified. We finally conclude in the last section.

\section{LOW SCALE SUSY FROM THE WARPED SPACETIME}

The RS spacetime has an extra dimension $y$ which is compactified in an $S_{1} / Z_{2}$ orbifold. Therefore $y$ takes values in the range $[-\pi, \pi]$, and $-y$ is identified with $y$. There are two orbifold fixed points at $y=0$ and $y=1$ where the UV and IR branes locate respectively. The solution to the Einstein's equation of such configuration is found to be a metric of an $\mathrm{AdS}_{5}$ space:

$$
d s^{2}=e^{-2 k R|y|} \eta_{\mu \nu} d x^{\mu} d x^{\nu}-R^{2} d y^{2},
$$

where $R$ and $k$ are the $S_{1}$ radius and the AdS curvature respectively. We assume that all the MSSM supermultiplets live in the bulk of the $\mathrm{AdS}_{5}$ spacetime. A hidden sector confined on the UV brane plays the role of breaking SUSY spontaneously. The Planck scales of the 5D theory and its effective $4 \mathrm{D}$ theory are related by the warp factor $e^{-k R \pi}$ as follows

$$
M_{P 4}^{2}=\frac{M_{P 5}^{3}}{k}\left(1-e^{-2 k R \pi}\right) .
$$


The superfield formalism [7] of 5D theories employs 5D supermultiplets which are functions of $\left(x^{\mu}, y, \theta, \bar{\theta}\right)$. A 5D field corresponds to an infinite tower of 4D fields in the Kaluza-Klein (KK) decomposition. The massless zero modes of those 5D fields are used to describe the MSSM fields when all symmetries are manifest. 5D gauge supermultiplets are used to describe gauge bosons and gauginos. Each one of this type contains a 4D-like vector supermultiplet:

$$
V(x, y, \theta)=-\theta \sigma^{\mu} \bar{\theta} A_{\mu}(x, y)-i \bar{\theta}^{2} \theta \lambda_{1}(x, y)+i \theta^{2} \bar{\theta} \bar{\lambda}_{1}(x, y)+\frac{1}{2} \bar{\theta}^{2} \theta^{2} D(x, y),
$$

and a 4D-like chiral supermultiplet:

$$
\chi(x, y, \theta)=\frac{1}{\sqrt{2}}\left(\Sigma(x, y)+i A_{5}(x, y)\right)+\sqrt{2} \theta \lambda_{2}(x, y)+\theta^{2} F_{\chi}(x, y) .
$$

They transform as an adjoint representation. The former is $Z_{2}$-even while the latter is $Z_{2}$-odd. The action for gauge supermultiplet is written as

$$
\begin{aligned}
S_{5}^{\text {gauge }}= & \int d^{5} x\left\{\frac{1}{4 g_{5}^{2}} \int d^{2} \theta R W^{\alpha} W_{\alpha}+\right.\text { h.c. } \\
& +\frac{2}{g_{5}^{2}} \int d^{4} \theta \frac{e^{-2 R \sigma}}{2 R} \operatorname{Tr}\left[\left\{e^{V / 2}, \partial_{5} e^{-V / 2}\right\}+\frac{1}{\sqrt{2}}\left(e^{V / 2} \chi^{\dagger} e^{-V / 2}+\left(e^{-V / 2} \chi e^{V / 2}\right)\right]^{2}\right\},
\end{aligned}
$$

in which the 5D gauge coupling $g_{5}$ relates to the $4 \mathrm{D}$ one $g_{4}$ as $g_{5}=\sqrt{2 \pi R} g_{4}$.

To describe the Higgs boson, matter fields, and their superpartners, 5D hypermultiplets are used . A hypermultiplet includes two 4D-like chiral superfields $\Phi$ and $\Phi^{c}$ which are in conjugate representations of a gauge group:

$$
\begin{aligned}
\Phi(x, y, \theta) & =\phi(x, y)+\sqrt{2} \theta \psi(x, y)+\theta^{2} F_{\Phi}(x, y), \\
\Phi^{c}(x, y, \theta) & =\phi^{c}(x, y)+\sqrt{2} \theta \psi^{c}(x, y)+\theta^{2} F_{\Phi^{c}}(x, y) .
\end{aligned}
$$

We assume that $\Phi$ is $Z_{2}$-even and $\Phi^{c}$ is $Z_{2}$-odd. The action for hypermultiplets and their gauge interaction reads:

$$
\begin{aligned}
S_{5}^{\text {matter }}= & \int d^{5} x\left\{\int d^{4} \theta R e^{-2 R \sigma}\left(\Phi^{\dagger} e^{-V} \Phi+\Phi^{c} e^{V} \Phi^{c \dagger}\right)\right. \\
& \left.+\int d^{2} \theta e^{-3 R \sigma} \Phi^{c}\left[\partial_{5}-\frac{1}{\sqrt{2}} \chi-\left(\frac{3}{2}-c_{\Phi}\right) R \sigma^{\prime}\right] \Phi+\text { h.c. }\right\},
\end{aligned}
$$

where $c_{\Phi}$ is a hypermultiplet bulk mass parameter. Solving the SUSY vacuum condition, we obtain the y-dependence of the massless zero mode of a hypermultiplet:

$$
\Phi(x, y, \theta)=\varphi_{0}(x, \theta) e^{\left(\frac{3}{2}-c_{\Phi}\right) R \sigma}+\ldots
$$

The Yukawa interactions between bulk hypermultiplets in 5D theory can be described by the following action:

$$
S_{5}^{\text {Yukawa }}=\int d^{5} x \int d^{2} \theta e^{-3 R \sigma} \frac{y_{123}}{M_{P 5}^{3 / 2}}[\delta(y)+\lambda \delta(y-\pi)] \Phi_{1} \Phi_{2} \Phi_{3} .
$$

To avoid the $\mu$-problem associating with the Higgs SUSY mass $\mu$ in the MSSM superpotential, we use the Giudice-Masiero mechanism [8], and imposing an $U(1)_{R^{-}}$symmetry for the theory. 
Table 1. R-charges of $\theta$ and MSSM hypermultiplets.

\begin{tabular}{|c|c|c|c|c|c|c|c|c|}
\hline$\theta$ & $Q_{i}^{h}$ & $U_{i}^{h}$ & $D_{i}^{h}$ & $L_{i}^{h}$ & $E_{i}^{h}$ & $H_{u}^{h}$ & $H_{d}^{h}$ & $X$ \\
\hline 1 & 1 & 1 & 1 & 1 & 1 & 0 & 0 & 0 \\
\hline
\end{tabular}

R-charges are assigned to $\theta$ and MSSM hypermultiplets as in Table 1. Under the action of Rsymmetry, the term $\mu H_{u}^{h} H_{d}^{h}$ only emerges after SUSY is broken in the hidden sector.

On the UV brane, the hidden sector superfield $X$ is assigned $\mathrm{R}$-charge 0 . Therefore the $\mathrm{R}$-charge of its F-term, $F_{X}$, is nonzero. Via some hidden dynamics, this F-term acquires nonzero vacuum expectation value (VEV), $\left\langle F_{X}\right\rangle$. The emergence of such a nonzero constant $\left\langle F_{X}\right\rangle$ explicitly breaks SUSY as well as R-symmetry. In gravity mediation, the SUSY breaking source is transmitted to the MSSM sector via contact terms in the action between the visible and hidden sectors suppressed by the Planck scale. These interactions result in the usual MSSM soft SUSY breaking terms.

The action that describes the interactions between gauge supermultiplets and the hidden field $X$ has the form:

$$
S_{5}^{X g}=\int d^{5} x\left[\int d^{2} \theta d_{a} \frac{X}{2 \pi R M_{P 5}^{2}} W^{a \alpha} W_{\alpha}^{a}+\text { h.c. }\right] \delta(y) .
$$

The contact terms between Higgs hypermultiplets and $X$ field are written as follows:

$$
\begin{aligned}
S_{5}^{X h}=\int d^{5} x \int d^{4} \theta & \left\{\left[d_{\mu} \frac{X^{\dagger}}{M_{P 5}^{2}} H_{u}^{h} H_{d}^{h}+d_{B_{\mu}} \frac{X^{\dagger} X}{M_{P 5}^{3}} H_{u}^{h} H_{d}^{h}+\text { h.c. }\right]\right. \\
+ & {\left[d_{A}^{H_{u}} \frac{X+X^{\dagger}}{M_{P 5}^{2}} H_{u}^{h^{\dagger}} H_{u}^{h}+d_{m}^{H_{u}} \frac{X^{\dagger} X}{M_{P 5}^{3}} H_{u}^{h \dagger} H_{u}^{h}\right.} \\
& \left.\left.+d_{A}^{H_{d}} \frac{X+X^{\dagger}}{M_{P 5}^{2}} H_{d}^{h^{\dagger}} H_{d}^{h}+d_{m}^{H_{d}} \frac{X^{\dagger} X}{M_{P 5}^{3}} H_{d}^{h^{\dagger}} H_{d}^{h}\right]\right\} \delta(y),
\end{aligned}
$$

and those between matter hypermultiplets and the hidden sector:

$$
S_{5}^{X m}=\int d^{5} x \int d^{4} \theta\left[\left(d_{A}^{\Phi}\right)_{i j} \frac{X+X^{\dagger}}{M_{P 5}^{2}} \Phi_{i}^{\dagger} \Phi_{j}+\left(d_{m}^{\Phi}\right)_{i j} \frac{X^{\dagger} X}{M_{P 5}^{3}} \Phi_{i}^{\dagger} \Phi_{j}\right] \delta(y),
$$

where $\Phi$ stands for $\left\{Q^{h}, U^{h}, D^{h}, L^{h}, E^{h}\right\}$ hypermultiplets, and $\{i, j\}$ are generation indices. The below contact terms correspond to 4-point couplings between the ordinary hypermultiplets and the hidden sector field:

$$
\begin{aligned}
S_{5}^{X a}=\int d^{5} x \int d^{2} \theta\{ & \frac{\left(a_{u}\right)_{i j}}{M_{P 5}^{5 / 2}} X H_{u}^{h} Q_{i}^{h} U_{j}^{h}+\frac{\left(a_{d}\right)_{i j}}{M_{P 5}^{5 / 2}} X H_{d}^{h} Q_{i}^{h} D_{j}^{h} \\
& \left.+\frac{\left(a_{e}\right)_{i j}}{M_{P 5}^{5 / 2}} X H_{d}^{h} L_{i}^{h} E_{j}^{h}+\text { h.c. }\right\} \delta(y) .
\end{aligned}
$$

After performing the integration over the 5th dimension, we obtain the $4 \mathrm{D}$ effective theory. All zero-modes of the bulk hypermultiplets are redefined to have $4 \mathrm{D}$ effective theory with 
canonical kinetic terms:

$$
\varphi_{0} \longrightarrow \mathscr{K}_{\Phi} \varphi_{0}
$$

where $\varphi_{0}$ represents the usual 4D MSSM fields, $\left\{H_{u}, H_{d}, Q_{i}, U_{i}, D_{i}, L_{i}, E_{i}\right\}$, and

$$
\mathscr{K}_{\Phi}=\sqrt{\frac{\left(1-2 c_{\Phi}\right) k}{2\left(e^{\left(1-2 c_{\Phi}\right) R k \pi}-1\right)}} .
$$

As a result of deriving the 4D effective theory, the 4D effective Yukawa couplings are now written in terms of 5D Yukawa couplings (10) and relevant bulk mass parameters:

$$
\begin{aligned}
\left(Y_{u}\right)_{i j} & =\frac{\left(y_{u}\right)_{i j}}{M_{5}^{3 / 2}}\left[1+\lambda e^{\left(\frac{3}{2}-c_{H u}-c_{Q i}-c_{Q j}\right) k R \pi}\right] \mathscr{K}_{H_{u}} \mathscr{K}_{Q_{i}} \mathscr{K}_{U_{j}}, \\
\left(Y_{d}\right)_{i j} & =\frac{\left(y_{d}\right)_{i j}}{M_{5}^{3 / 2}}\left[1+\lambda e^{\left(\frac{3}{2}-c_{H d}-c_{Q i}-c_{D j}\right) k R \pi}\right] \mathscr{K}_{H_{d}} \mathscr{K}_{Q_{i}} \mathscr{K}_{D_{j}}, \\
\left(Y_{e}\right)_{i j} & =\frac{\left(y_{e}\right)_{i j}}{M_{5}^{3 / 2}}\left[1+\lambda e^{\left(\frac{3}{2}-c_{H d}-c_{L i}-c_{E j}\right) k R \pi}\right] \mathscr{K}_{H_{d}} \mathscr{K}_{L_{i}} \mathscr{K}_{E_{j}} .
\end{aligned}
$$

The 4D effective actions containing the MSSM soft SUSY breaking terms can be derived from Eqs. (11), (12), (13), and (14). After the $X$ field gets its VEV $\langle X\rangle=\theta^{2}\left\langle F_{X}\right\rangle$, the MSSM soft SUSY breaking terms emerge automatically in the Lagrangian. As a consequence of that calculation, gaugino masses are derived from Eq. (11) as

$$
M_{a}=-d_{a} g_{a}^{2} \frac{\left\langle F_{X}\right\rangle}{2 \pi R M_{P 5}^{2}}, \quad(a=1,2,3) .
$$

Eq. (13) leads to scalar soft masses of sparticles:

$$
\left(m_{\Phi}\right)_{i j}^{2}=-\left(d_{m}^{\Phi}\right)_{i j} \frac{\left|\left\langle F_{X}\right\rangle\right|^{2}}{M_{P 5}^{3}} \mathscr{K}_{\Phi_{i}} \mathscr{K}_{\Phi_{j}}+\sum_{n=1}^{3} \frac{\left|\left\langle F_{X}\right\rangle\right|^{2}}{M_{P 5}^{4}}\left(d_{A}^{\Phi}\right)_{i n}\left(d_{A}^{\Phi}\right)_{n j} \mathscr{K}_{\Phi_{n}}^{2} \mathscr{K}_{\Phi_{i}} \mathscr{K}_{\Phi_{j}},
$$

where $\Phi$ stands for $Q, U, D, L, E$, and $i, j=\{1,2,3\}$. Soft masses of the Higgs sector can be obtained from (12) as

$$
\begin{aligned}
m_{H_{u}}^{2} & =-d_{m}^{H_{u}} \frac{\left|\left\langle F_{X}\right\rangle\right|^{2}}{M_{P 5}^{3}} \mathscr{K}_{H_{u}}^{2}+\frac{\left|\left\langle F_{X}\right\rangle\right|^{2}}{M_{P 5}^{4}}\left(d_{A}^{H_{u}}\right)^{2} \mathscr{K}_{H_{u}}^{4}, \\
m_{H_{d}}^{2} & =-d_{m}^{H_{d}} \frac{\left|\left\langle F_{X}\right\rangle\right|^{2}}{M_{P 5}^{3}} \mathscr{K}_{H_{d}}^{2}+\frac{\left|\left\langle F_{X}\right\rangle\right|^{2}}{M_{P 5}^{4}}\left(d_{A}^{H_{d}}\right)^{2} \mathscr{K}_{H_{d}}^{4}, \\
B_{\mu} & =d_{B_{\mu}} \frac{\left|\left\langle F_{X}\right\rangle\right|^{2}}{M_{P 5}^{3}} \mathscr{K}_{H_{u}} \mathscr{K}_{H_{d}}-d_{A}^{H_{u}} \frac{\left\langle F_{X}\right\rangle \mid}{M_{P 5}^{2}} \mu \mathscr{K}_{H_{u}}^{2}-d_{A}^{H_{d}} \frac{\left|\left\langle F_{X}\right\rangle\right|}{M_{P 5}^{2}} \mu \mathscr{K}_{H_{d}}^{2} .
\end{aligned}
$$

As mentioned above, the ordinary SUSY Higgs mass originates from SUSY breaking source $\left\langle F_{X}\right\rangle$ in the first term of Eq. (12) just as other soft SUSY breaking terms:

$$
\mu=d_{\mu} \frac{\left\langle F_{X}\right\rangle^{\dagger}}{M_{P 5}^{2}} \mathscr{K}_{H_{u}} \mathscr{K}_{H_{d}}
$$


Similarly, the trilinear couplings A-terms come from Eq. (14) as follows

$$
\begin{aligned}
\left(A_{u}\right)_{i j}= & \frac{1}{\left(Y_{u}\right)_{i j}}\left\{\left(a_{u}\right)_{i j} \frac{\left\langle F_{X}\right\rangle}{M_{P 5}^{5 / 2} \mathscr{K}_{H_{u}} \mathscr{K}_{Q_{i}} \mathscr{K}_{Q_{j}}}\right. \\
& \left.-\left[d_{A}^{H_{u}}\left(Y_{u}\right)_{i j} \mathscr{K}_{H_{u}}^{2}+\sum_{n=1}^{3}\left(d_{A}^{Q}\right)_{n i}\left(Y_{u}\right)_{n j} \mathscr{K}_{Q_{n}} \mathscr{K}_{Q_{i}}+\sum_{n=1}^{3}\left(d_{A}^{U}\right)_{n j}\left(Y_{u}\right)_{i n} \mathscr{K}_{U_{n}} \mathscr{K}_{U_{j}}\right] \frac{\left\langle F_{X}\right\rangle}{M_{P 5}^{2}}\right\}, \\
\left(A_{d}\right)_{i j}= & \frac{1}{\left(Y_{d}\right)_{i j}}\left\{\left(a_{d}\right)_{i j} \frac{\left\langle F_{X}\right\rangle}{M_{P 5}^{5 / 2}} \mathscr{K}_{H_{d}} \mathscr{K}_{Q_{i}} \mathscr{K}_{D_{j}}\right. \\
& \left.-\left[d_{A}^{H_{d}}\left(Y_{d}\right)_{i j} \mathscr{K}_{H_{d}}^{2}+\sum_{n=1}^{3}\left(d_{A}^{Q}\right)_{n i}\left(Y_{d}\right)_{n j} \mathscr{K}_{Q_{n}} \mathscr{K}_{Q_{i}}+\sum_{n=1}^{3}\left(d_{A}^{D}\right)_{n j}\left(Y_{d}\right)_{i n} \mathscr{K}_{D_{n}} \mathscr{K}_{D_{j}}\right] \frac{\left\langle F_{X}\right\rangle}{M_{P 5}^{2}}\right\}, \\
\left(A_{e}\right)_{i j}= & \frac{1}{\left(Y_{e}\right)_{i j}}\left\{\left(a_{d}\right)_{i j} \frac{\left\langle F_{X}\right\rangle}{M_{P 5}^{5 / 2}} \mathscr{K}_{H_{d}} \mathscr{K}_{L_{i}} \mathscr{K}_{E_{j}}\right. \\
& \left.-\left[d_{A}^{H_{d}}\left(Y_{e}\right)_{i j} \mathscr{K}_{H_{d}}^{2}+\sum_{n=1}^{3}\left(d_{A}^{L}\right)_{n i}\left(Y_{e}\right)_{n j} \mathscr{K}_{L_{n}} \mathscr{K}_{L_{i}}+\sum_{n=1}^{3}\left(d_{A}^{E}\right)_{n j}\left(Y_{e}\right)_{i n} \mathscr{K}_{E_{n}} \mathscr{K}_{E_{j}}\right] \frac{\left\langle F_{X}\right\rangle}{M_{P 5}^{2}}\right\}
\end{aligned}
$$

\section{IMPLICATION OF THE HIGGS MASS MEASUREMENT}

Due to the effect of the warp factor, $e^{-k R \pi}$, the KK scale which is originally proportional to the compactification scale in usual theories is warped down exponentially to a much lower scale, $k e^{-k R \pi}$. In our analysis, we use the KK scale as the cutoff scale of the effective theory, $M_{\text {cut }}=k e^{-k R \pi} \simeq \mathscr{O}(100) \mathrm{TeV}$, where the boundary conditions are set. Since the renormalization group running distance from the cutoff scale toward $\mathrm{TeV}$ scale (or the electroweak scale) is very short, we can safely neglect such running effect, and approximately determine the soft SUSY breaking terms as those defined at the boundary.

The SM-like Higgs boson mass of the model is determined at one-loop corrections [10] as a function of the stop mixing parameter $X_{t}=A_{t}-\mu \cot \beta$, the mixing angle $\beta$ between the VEVs of the two Higgs doublets, and the average stop mass $m_{\tilde{t}}{ }^{1}$. Within the above approximation, the trilinear couling $A_{t}$ and the average stop mass $m_{\tilde{t}}$ are defined at the boundary as input parameters. Their values can be determined from the underlying 5D theory using Eqs. (26) and (21). We use the combined result of the SM-like Higgs boson mass measurement at ATLAS and CMS experiment [9],

$$
m_{h}=125.09 \pm 0.24 \mathrm{GeV}
$$

\footnotetext{
${ }^{1}$ The sfermion loop corrections to the Higgs mass are proportional to the corresponding Yukawa couplings. Therefore, the dominant loop contribution comes from the stop due to its strong Yukawa coupling. Contributions from other sfermions are negligible.
} 
to set the $5 \sigma$ constrained limits of the SM-like Higgs boson mass in our study:

$$
123.89 \lesssim m_{h} \lesssim 126.29 \quad(\mathrm{GeV})
$$

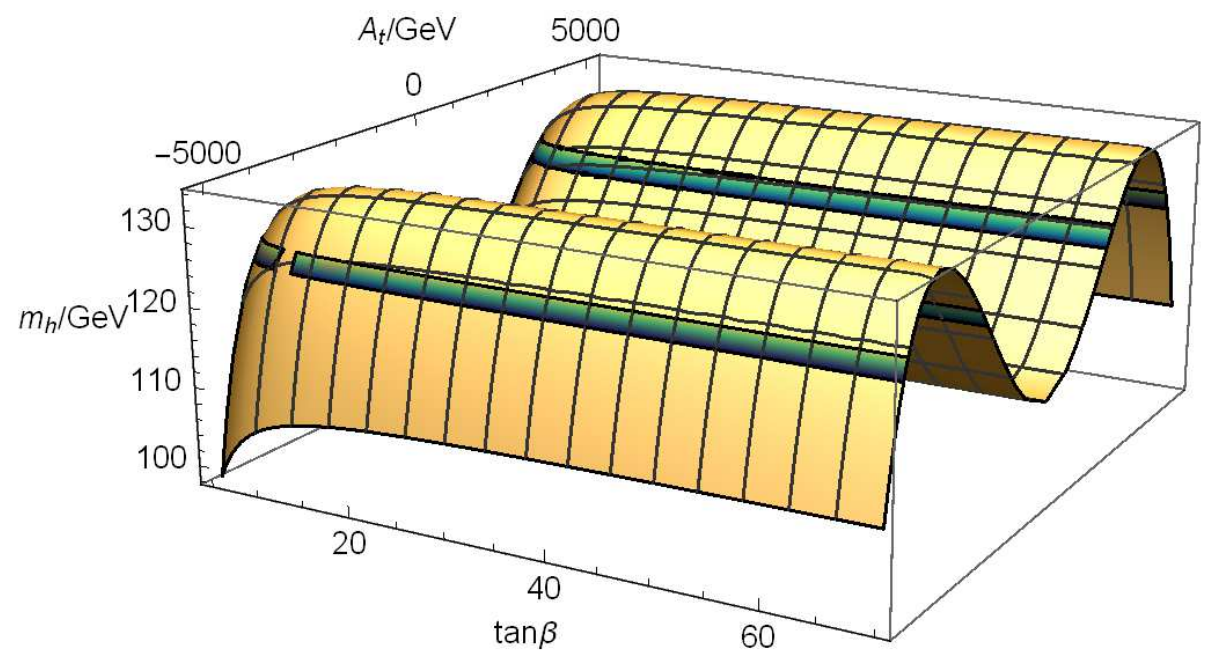

Fig. 1. The Higgs boson mass as a function of $\left(\tan \beta, A_{t}\right)$. In this case, $\mu=500 \mathrm{GeV}$ and $m_{\tilde{t}}=1500 \mathrm{GeV}$.

Here we are interested in the mild $\mu$ case in order to avoid reintroducing fine-tuning while ensuring Higgsino masses satisfy the current LHC lower limit. Therefore, $\mu=500 \mathrm{GeV}$ is fixed in throughout the analysis. Choosing $m_{\tilde{t}}=1.5 \mathrm{TeV}$, in Fig. 1 we show the plot of the SM-like Higgs boson mass as a function of $\tan \beta$ and $A_{t}$. The black/green region correspond to the LHC limits (30). In order to identify the viable parameter region that can reproduce such Higgs boson mass, we project that green region onto the $\left(\tan \beta, A_{t}\right)$ plane. The result is shown as black/green strips in Fig. 2. We find that for the above choice of stop mass, most of the parameter region is excluded by the Higgs mass constraint, only the narrow strips still survive. The allowed regions for the trilinear coupling $A_{t}$ in this case locate around $\pm 2250 \mathrm{GeV}$ and $\pm 4700 \mathrm{GeV}$.

Similarly, we plot the Higgs boson mass as functions of $\left(\tan \beta, m_{\tilde{t}}\right)$ and $\left(A_{t}, m_{\tilde{t}}\right)$ in Figs. 3 and 5 respectively. The color codes are the same as above. The corresponding viable parameter regions are identified as black/green areas in Figs. 4 and 6. In Fig. 6, we see that for $A_{t} \sim \pm 3$ $\mathrm{TeV}$, the stop mass is around $2 \mathrm{TeV}$. This small parameter region can be tested in the future run of the LHC. In Figs. 4 and 6, we find that the region with a large stop mass $m_{\tilde{t}}=5-13 \mathrm{TeV}$ is more favored in terms of the Higgs mass constraint. This region is actually beyond the reach of the LHC $14 \mathrm{TeV}$. We observe that the Higgs boson mass measurement limits the stop mass to be less than $20 \mathrm{TeV}$ in most of the parameter space. Such mass range can be probed at a future 100 $\mathrm{TeV}$ hardon collider via stop pair production channels. 


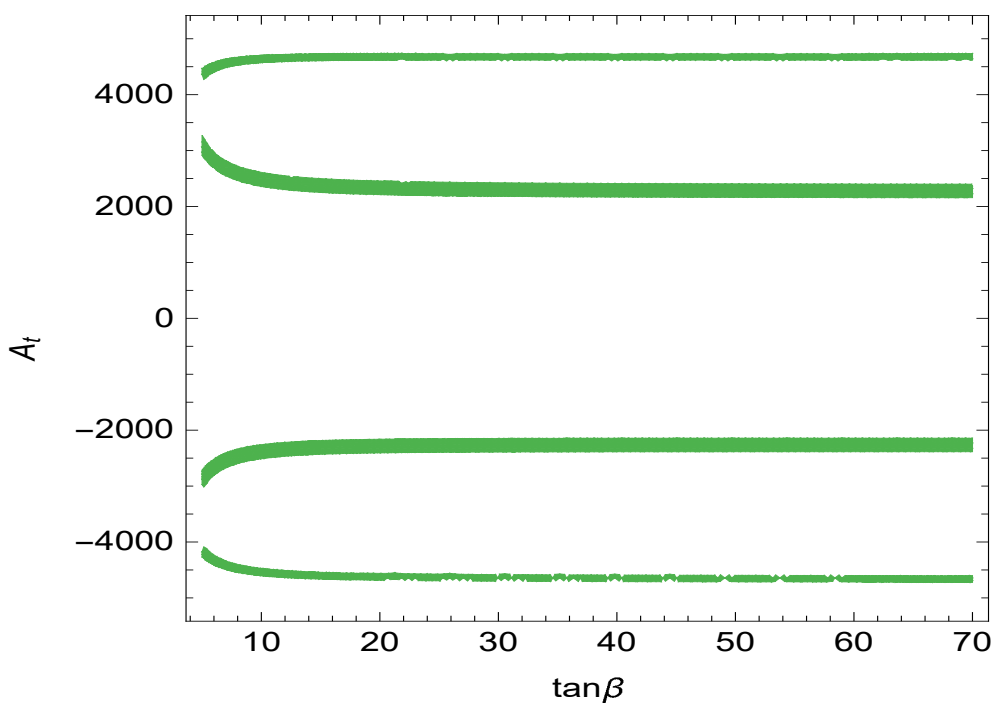

Fig. 2. Parameter region in the plane $\left(\tan \beta, A_{t}\right)$ with $\mu=500 \mathrm{GeV}$ and $m_{\tilde{t}}=1500 \mathrm{GeV}$ that can reproduce the Higgs boson mass measured at the LHC (Eq. (30)).

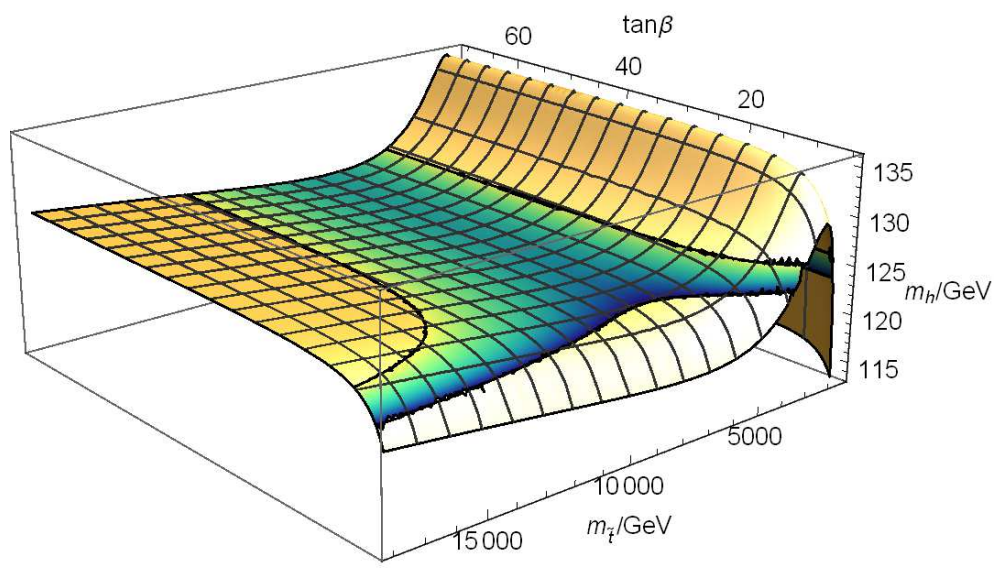

Fig. 3. The Higgs boson mass as a function of $\left(\tan \beta, m_{\tilde{t}}\right)$. In this case, $\mu=500 \mathrm{GeV}$ and $A_{t}=5000 \mathrm{GeV}$. 


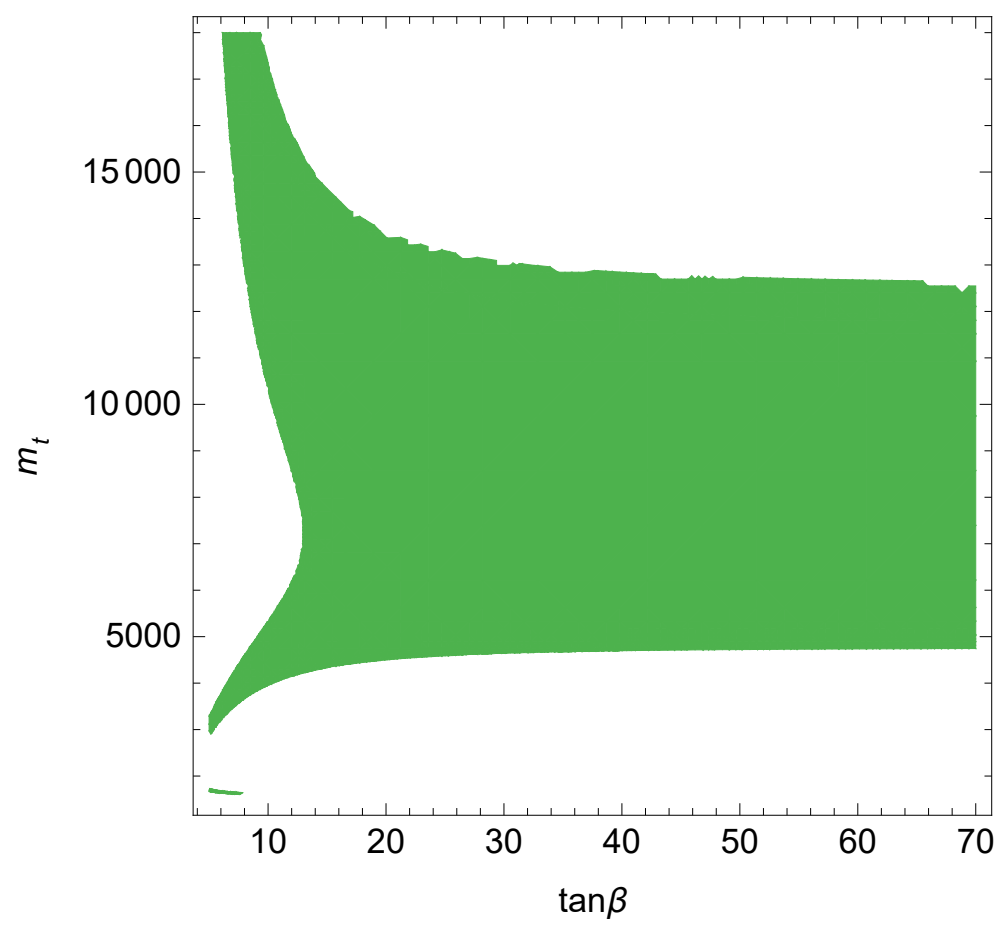

Fig. 4. Parameter region in the plane $\left(\tan \beta, m_{\tilde{t}}\right)$ with $\mu=500 \mathrm{GeV}$ and $A_{t}=5000 \mathrm{GeV}$ that can reproduce the Higgs boson mass measured at the LHC (Eq. (30)).

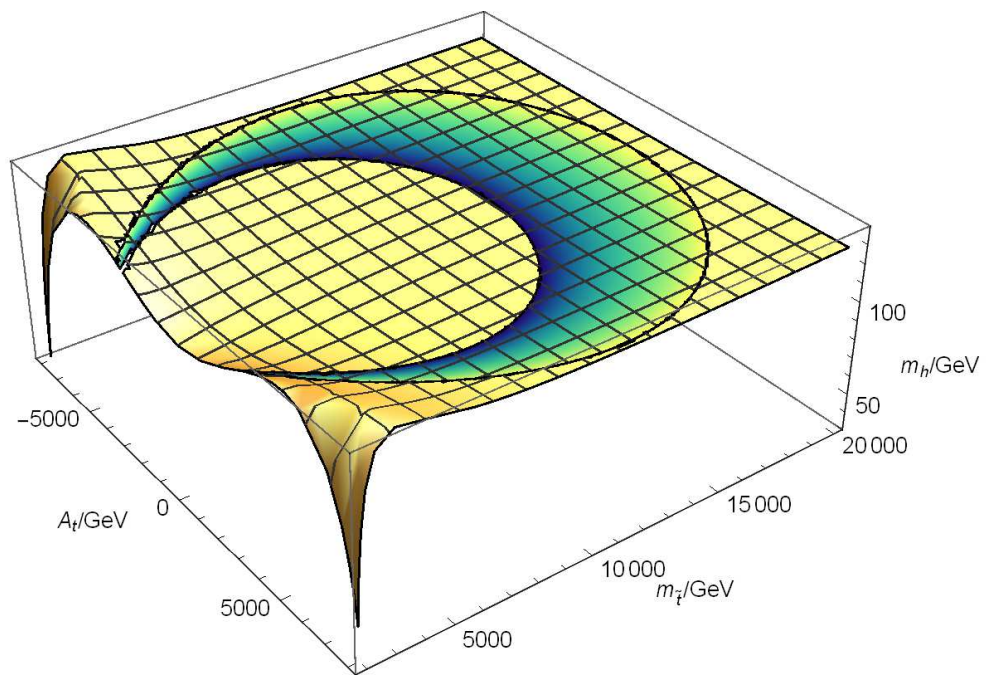

Fig. 5. The Higgs boson mass as a function of $\left(A_{t}, m_{\tilde{t}}\right)$. In this case, $\mu=500 \mathrm{GeV}$ and $\tan \beta=10$. 


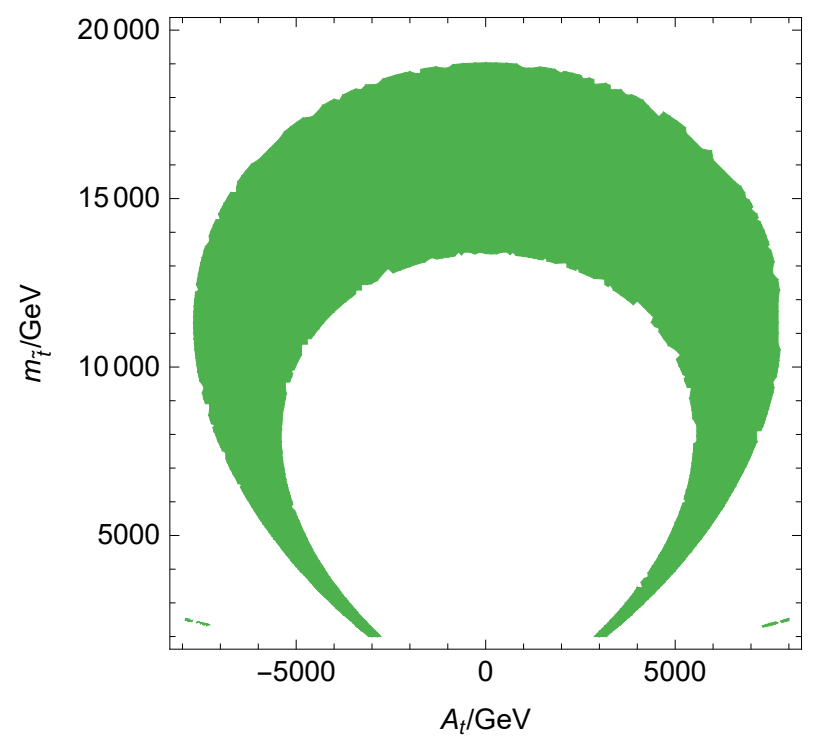

Fig. 6. Parameter region in the plane $\left(A_{t}, m_{\tilde{t}}\right)$ with $\mu=500 \mathrm{GeV}$ and $\tan \beta=10$ that can reproduce the Higgs boson mass measured at the LHC (Eq. (30)).

\section{CONCLUSION}

The Higgs boson discovery has not only fulfilled the particle contents of the SM, but also set a severe constraint on many models of physics beyond the SM via the Higgs mass measurement at the LHC. Here, we have investigated a SUSY model in the RS background. The hidden sector which breaks SUSY spontaneously is confined on the UV brane, while all the MSSM superfields live in the bulk. The RS background naturally brings the high energy scale toward the low energy scale thanks to the warp factor. Therefore the cutoff scale can be much lower than usual. Within this setup, the 4D effective soft couplings and masses has been derived from the original 5D Lagrangian. We have examined the constraint of the Higgs mass measurement at the LHC on the parameter space of the model, and identified the viable parameter regions that can reproduce a 125 GeV SM-like Higgs boson. According to that, there are small regions testable at the future run of the LHC where the average stop mass is around $2 \mathrm{TeV}$. Nevertheless, most of the allowed region is beyond the reach of the LHC. However, the Higgs mass constraint also rules out a dominant region with the stop mass larger than $20 \mathrm{TeV}$. This fact implies that a future $100 \mathrm{TeV}$ hardron collider can test the model's viability.

\section{Acknowledgment}

This work is funded by Vietnam National Foundation for Science and Technology Development (NAFOSTED) under the grant No. 103.01-2014.22.

\section{REFERENCES}

[1] G. Aad et al. [ATLAS Collaboration], Phys. Lett. B, 716 (2012) 1 [arXiv:1207.7214 [hep-ex]]; S. Chatrchyan et al. [CMS Collaboration], Phys. Lett. B, 716 (2012) 30 [arXiv:1207.7235 [hep-ex]]. 
[2] See for example: I. G. Garcia, K. Howe and J. March-Russell, JHEP, 1512 (2015) 005 [arXiv:1510.07045 [hepph]]; Z. Lalak, M. Lewicki, M. McGarrie and P. Olszewski, JHEP, 1511 (2015) 137 [arXiv:1508.05105 [hep-ph]]; M. Asano, Y. Nakai and N. Yokozaki, Phys. Rev. D, 93 (2016) no.5, 055023 [arXiv:1512.02201 [hep-ph]].

[3] T. Gherghetta and A. Pomarol, Nucl. Phys. B 586, 141 (2000) [hep-ph/0003129]; T. Gherghetta and A. Pomarol, Nucl. Phys. B 602, 3 (2001) [hep-ph/0012378]; S. J. Huber and Q. Shafi, Phys. Lett. B 498, 256 (2001) [hepph/0010195]; H. Davoudiasl, J. L. Hewett and T. G. Rizzo, Phys. Lett. B 493, 135 (2000) [hep-ph/0006097]; H. Davoudiasl, J. L. Hewett and T. G. Rizzo, Phys. Lett. B 473, 43 (2000) [hep-ph/9911262]; S. J. Huber and Q. Shafi, Phys. Rev. D 63, 045010 (2001) [hep-ph/0005286]; Y. Grossman and M. Neubert, Phys. Lett. B 474, 361 (2000) [hep-ph/9912408]; G. R. Dvali and M. A. Shifman, Phys. Lett. B 475, 295 (2000) [hep-ph/0001072]; A. Masiero, M. Peloso, L. Sorbo and R. Tabbash, Phys. Rev. D 62, 063515 (2000) [hep-ph/0003312].

[4] N. Okada and T. Yamada, Phys. Rev. D, 84 (2011) 035005 [arXiv:1105.0241 [hep-ph]]; N. Okada and H. M. Tran, Phys. Rev. D, 94 (2016) no.7, 075016 [arXiv:1606.05329 [hep-ph]].

[5] L. Randall and R. Sundrum, Phys. Rev. Lett., 83 (1999) 3370 [hep-ph/9905221].

[6] See for example: C. Han, T. T. Yanagida and N. Yokozaki, Phys. Rev. D, 93 (2016) no.5, 055025 [arXiv:1602.04204 [hep-ph]]; A. Abdalgabar, A. S. Cornell, A. Deandrea and M. McGarrie, JHEP, 1601 (2016) 083 [arXiv:1504.07749 [hep-ph]]; T. Gherghetta, B. von Harling, A. D. Medina, M. A. Schmidt and T. Trott, Phys. Rev. D, 91 (2015) 105004 [arXiv:1502.07173 [hep-ph]]; E. Hardy, J. March-Russell and J. Unwin, JHEP, 1210 (2012) 072 [arXiv:1207.1435 [hep-ph]].

[7] N. Arkani-Hamed, T. Gregoire and J. G. Wacker, JHEP 0203, 055 (2002) [hep-th/0101233]; M. A. Luty and R. Sundrum, Phys. Rev. D 64, 065012 (2001) [hep-th/0012158]; J. Bagger and D. V. Belyaev, Phys. Rev. D 67, 025004 (2003) [hep-th/0206024]; D. Marti and A. Pomarol, Phys. Rev. D 64, 105025 (2001) [hep-th/0106256].

[8] G. F. Giudice and A. Masiero, Phys. Lett. B, 206 (1988) 480.

[9] G. Aad et al. [ATLAS and CMS Collaborations], Phys. Rev. Lett., 114 (2015) 191803 [arXiv:1503.07589 [hepex]].

[10] H. E. Haber, R. Hempfling and A. H. Hoang, Z. Phys. C, 75 (1997) 539 [hep-ph/9609331]; A. Djouadi, Phys. Rept., 459 (2008) 1 [hep-ph/0503173]. 